\title{
MicroRNA-144-3p suppresses tumor growth and angiogenesis by targeting SGK3 in hepatocellular carcinoma
}

\author{
MANYA WU ${ }^{1}$, CHAOYUAN HUANG $^{2}$, XINPING HUANG ${ }^{1}$, \\ RONG LIANG ${ }^{3}$, YAN FENG ${ }^{1}$ and XIAOLING LUO ${ }^{1}$ \\ ${ }^{1}$ Research Department, ${ }^{2}$ Department of Hepatobiliary Surgery and ${ }^{3}$ First Department of Chemotherapy, \\ Affiliated Tumor Hospital of Guangxi Medical University, Nanning, \\ Guangxi Zhuang Autonomous Region 530021, P.R. China
}

Received March 7, 2017; Accepted August 3, 2017

DOI: $10.3892 /$ or.2017.5900

\begin{abstract}
In our previous studies, the Illumine Soledad massively parallel signature sequencing of miRNomes in non-tumor and hepatocellular carcinoma (HCC) tissues revealed that microRNA (miR)-144-3p was significantly downregulated in $\mathrm{HCC}$, but its role in HCC development, especially angiogenesis, remains unclear. In this investigation, we found recovering miR-144-3p expression can significantly suppress the growth, migration and induced angiogenic capacity of HCC cells through both in vivo and in vitro experiments. Moreover, clinical correlation analysis showed that low expression of miR-144-3p was positively correlated to poor disease-free survival (DFS) of HCC patients. Mechanistically, serum and glucocorticoid kinase 3 (SGK3), the putative targets of miR-144-3p, was predicted by Target Scan database and identified to be suppressed by miR-144-3p so that inhibiting the activation of mTOR-VEGF downstream signals was activated by the phosphoinositide 3-kinase (PI3K)-independent pathway. Hence, we concluded that miR-144-3p, which is frequently downregulated in HCC, can inhibit proliferation, migration and repress angiogenesis by regulating SGK3 activation with $\mathrm{PI} 3 \mathrm{~K}$ independent signal pathway, and acts as a prognostic factor for HCC patients.
\end{abstract}

Correspondence to: Professor Xiaoling Luo, Research Department, Affiliated Tumor Hospital of Guangxi Medical University, 71 Hedi Road, Nanning, Guangxi Zhuang Autonomous Region 530021, P.R. China

E-mail: luoxiaoling67@126.com

Abbreviations: HCC, hepatocellular carcinoma; SGK3, serum and glucocorticoid kinase 3; PI3K, phosphoinositide 3-kinase; miRNAs, microRNAs; ZEB1, zinc finger E-box-binding homeobox 1; ZEB2, zinc finger E-box-binding homeobox 2; E2F3, E2F transcription factor 3; ZFX, zinc finger X-chromosomal protein; VEGF, vascular endothelial growth factor; $\mathrm{NC}$, negative control

Key words: hepatocellular carcinoma, microRNA-144-3p, serum and glucocorticoid kinase 3 , growth, prognosis, angiogenesis

\section{Introduction}

Hepatocellular carcinoma (HCC) is one of the most fatal cancers in the world while its incidence and death rate are still increasing (1). Most studies have demonstrated that HCC is a complex and multifarious molecular disease $(2,3)$. However, the molecular pathogenesis of $\mathrm{HCC}$ remains elusive. Elucidation of some aberrant genes and mechanisms in HCC is required to investigate more specific potent targeted therapy and diagnosis methods.

MicroRNAs (miRNAs) are a type of small and endogenous non-coding RNAs of 19-23 nucleotides that negatively regulate gene expression by binding to the untranslated regions of target messenger RNAs (mRNAs). Growing evidence indicates that miRNAs play an important role in diverse biological processes, and the aberrant expression of miRNAs contributes to tumorigenesis, progression, diagnosis and prognosis (4-6). miR-144-3p has been reported aberrantly and lowly expressed in thyroid cancer targeting ZEB1 and ZEB2 could suppress the invasion and migration capability of thyroid cancer cells (7). Moreover, as it exerts direct regulatory roles on ZFX expression, further investigations showed that miR-144-3p expression not only inhibits NSCLC tumor cell growth but induces apoptosis (8). Similar to previous studies, our previous microarray profiling also found that miR-144-3p was downregulated in HCC (9). Though several reports revealed that miR-144-3p can suppress proliferation of HCC by targeting AKT3 and E2F3 (10,11), the function of miR-144-3p in tumor angiogenesis is still unknown $(12,13)$, hence we investigated the role of miR-144-3p in angiogenesis and the relationship with the survival time of HCC patients so that we can explore new targets of anti-angiogenesis treatments in clinic.

In this study, we found miR-144-3p not only suppressed tumor growth in HCC, but also inhibited the ability of angiogenesis of HCC cells. Functional studies also demonstrated that the inhibitory effect of miR-144-3p on HCC is mainly mediated by targeted SGK3, which reduces the activity of mTOR-VEGF pathway in PI3K downstream signaling.

\section{Materials and methods}

Patients and tissue samples. Surgically resected paired HCC and adjacent non-cancerous tissues were collected from 
51 primary HCC patients at the Affiliated Tumor Hospital of Guangxi Medical University between March 2011 and May 2013. Tissue samples were immediately frozen in liquid nitrogen until analysis. These cases selected were based on a clear pathological diagnosis, follow-up data, and had first undergone radical resection of HCC, and had not received preoperative adjuvant chemotherapy, radiotherapy, targeted therapy or immunotherapy. Informed consent was obtained from each patient, and the study was approved by the Ethics Committee of Guangxi Medical University, Nanning, China. The investigations were conducted according to the Principles of Declaration of Helsinki.

Cell culture and transfection. All cells were obtained from the Institute of Biochemistry and Cell Biology of Chinese Academy of Science (Shanghai, China). Human HCC cell lines (QGY-7703, SK-hep1 and human normal liver cells (HL-7702) were maintained in RPMI-1640 with $10 \%$ fetal bovine serum (FBS) (Gibco, USA) at $37^{\circ} \mathrm{C}$ in a humidified incubator containing 5\% $\mathrm{CO}_{2}$. miR-144-3p duplex mimics and negative control (NC) were from GenePharma (Shanghai, China). Sequences used for the miR-144-3p mimics and the corresponding control were 5'-UACAGUAUAGAUGAUG UACU-3' and 5'-UUUGUACUACACAAAAGUACUG-3', respectively. Sequences used for SGK3 siRNA, and NC were 5'-GCAUUGGGUUACAUUTT-3', and 5'-UGACCUCAACU ACAUGGUUTT-3', respectively. Cells were transfected with RNAs using INTERFERin ${ }^{\circledR}$ transfection reagent (Polyplus Transfection, Illkirch, France) at a final concentration of $100 \mathrm{nM}$ according to the manufacturer's instructions.

RNA extraction and quantitative RT-PCR. Total RNA, including miRNA, was extracted using TRIzol reagent (Invitrogen, CA, USA) according to the manufacturer's instructions. cDNAs were synthesized using ReverTra Ace ${ }^{\circledR}$ qPCR RT kit (FSQ-101, Toyobo, Japan). MicroRNA was reversely transcribed using First Strand cDNA Synthesis kit ReverTra Ace- $\alpha$ - (FSK-100, Toyobo). Real-time PCR analyses were performed with Thunderbird SYBR ${ }^{\circledR}$ qPCR Mix (QPS-201, Toyobo) on an MxPro-Mx3000P Sequence Detection system (Stratagene, USA). U6 small nuclear RNA was used as an internal normalized reference, and fold changes were calculated by relative quantification $\left(2^{-\Delta \Delta \mathrm{C} t}\right)$. The primers used were as follows: miR-144-3p specific stem-loop reverse transcription primers: 5'-GTCGTATCCAGTGCAGGGTCCG AGGTATTCGCACTGGATACGACAGTACA-3'; miR-144-3p forward, 5'-GGGAGATCAGAAGGTGATT-3'; reverse, 5'-GTG CAGGGTCCGAGGT-3'. U6 forward, 5'-CTCGCTTCGG CAGCACA-3'; reverse, 5'-AACGCTTCACGAATTTGCGT-3'. SGK3 forward, 5'-CCAGGAGTGAGTCTTACAG-3'; reverse, 5'-CCAGCCACATTAGGATTA-3'. All samples were amplified in triplicate according to the manufacturer's instructions.

Cell proliferation and colony formation assays. Cells were seeded into 96 -well plate $\left(5 \times 10^{3} /\right.$ well) and transfected with miR-144-3p mimics or NC. The cell proliferation of HCC cell lines was determined by way of Cell Counting Kit- 8 assay (Dojindo, Japan) at the indicated time points $(0,24,48$ and $72 \mathrm{~h}$ ) according to the manufacturer's instructions. The groups were carried out in quintuplicate wells. For colony formation assay, cells were seeded into 6-well plates at a low density $\left(1 \times 10^{3}\right.$ cells/per well) and cultured for 10 days. Then cells were fixed with $4 \%$ paraformaldehyde for $30 \mathrm{~min}$ and surviving colonies ( $>50$ cells/colony) were counted after staining with $1 \%$ crystal violet. The experiments were carried out in triplicate wells.

Cell cycle distribution. Forty-eight hours after transfection in 6-well plates, QGY-7703 or SK-hep1 were harvested and washed with cold 1X PBS. Then, cells were fixed in $70 \%$ ethanol at $4^{\circ} \mathrm{C}$ overnight, and washed with PBS twice, resuspended with $100 \mu 1$ RNase A, incubated at $37^{\circ} \mathrm{C}$ for 30 min. Staining for DNA content was performed with $400 \mu \mathrm{l}$ propidium iodide (KeyGen, Nanjing, China) at $4^{\circ} \mathrm{C}$ for $30 \mathrm{~min}$ in the dark, and analyzed by a flow cytometer (Beckman Coulter EPICS XL, USA). The experiments were carried out at least three times.

In vitro migration assay. Migration assays were performed using the 24-well Cell Migration with 8- $\mu$ m pore size polycarbonate membrane (Corning, NY, USA), according to the manufacturer's instructions. Briefly, 24 hours after the transfection, $5 \times 10^{4}$ cells were resuspended in $200 \mu 1$ serum-free medium and plated in the top chamber. The lower chambers were filled with $0.6 \mathrm{ml}$ of medium containing $10 \%$ FBS. Medium with $10 \%$ FBS was added to the lower chamber as a chemoattractant. After $24-\mathrm{h}$ incubation at $37^{\circ} \mathrm{C}$, the cells on the upper surface of the membrane were removed, and the cells on the lower surface were fixed, stained, photographed, and counted under a microscope in five fields.

In vitro capillary tube formation assay. HUVECs were cultured at $37^{\circ} \mathrm{C}$ for $24 \mathrm{~h}$ in a 96 -well plate coated with Matrigel (BD Biosciences, Bedford, MA, USA) in the absence or presence of culture medium of HCC cells transfected with NC or siRNA. The formation of capillary-like structures was captured under an inverted phase contrast microscope. The number of the formed tubes, which represent the degree of angiogenesis in vitro, were scanned and quantitated in five low power fields.

Animal studies. For mouse studies, male BALB/c-nude mice (5-6 weeks of age) were obtained from Guangxi Province Laboratory Animal Center (Nanning, China). HCC cells $\left(5 \times 10^{6}\right)$ after transfected with miRNA-mimics or NC were suspended in $0.1 \mathrm{ml}$ phosphate-buffered saline (PBS) and then injected subcutaneously into the backs of the mice. Tumor growth was measured over the course of 30 days, and tumor volume was calculated according to the formula: volume $=0.5 \mathrm{x}$ length $\mathrm{x}$ width ${ }^{2}$. All experiments were performed in accordance with the Guide for the Care and Use of Laboratory Animals, with the approval of the Guangxi medical University, China.

Luciferase reporter assay. SGK3-3'UTR-Mutant on binding sites was synthesized by PCR. HEK293T cells were co-transfected with $80 \mathrm{ng}$ of luciferase reporter plasmid and $40 \mathrm{ng}$ of pRL-TK-Renilla-luciferase plasmid (Promega, Madison, WI, USA), and the total concentration of RNA (100 nM) was detected with jetSI-ENDO transfection reagents (Polyplus 
Transfection). With incubation for $24 \mathrm{~h}$, luciferase activity was evaluated with the Dual-Luciferase Reporter assay system (Promega), and data were normalized for transfection efficiency via dividing firefly luciferase activity by that of Renilla luciferase.

Western blot analysis. Targeted prediction of miR-144-3p was analyzed by Target Scan. Antibodies for SKG3, p-PI3K, mTOR, VEGFR2, and $\beta$-actin were purchased from Cell Signaling Technology, and all the antibodies were rabbit anti-human. Cells were harvested and then lysed with RIPA buffer supplemented with $1 \mathrm{mmol} / 1 \mathrm{PMSF}$ (both from Boster, Wuhan, China), and then centrifuged at $14,000 \mathrm{x} \mathrm{g}$; at $4^{\circ} \mathrm{C}$ for $10 \mathrm{~min}$. Protein concentrations of the extracts were measured using the bicinchoninic acid (BCA) protein assay kit (KeyGen). Equal amounts of the proteins were concentrated and separated through SDS-PAGE, and then transferred to polyvinylidene difluoride (PVDF) membranes (Boster). After blocking in TBST (Tris-buffered saline with Tween-20) which contained 5\% non-fat milk for $60 \mathrm{~min}$, the membranes were incubated with the primary antibody (1:1,000 dilution; $\beta$-actin, as a loading control, 1:2,500 dilution) overnight at $4^{\circ} \mathrm{C}$. The membranes were incubated with the secondary antibodies (mouse anti-rabbit and HRP-linked antibody, 1:5,000 dilution; Cell Signaling Technology). After incubating in enhanced chemiluminescence solution (Boster), the proteins on the membranes were detected using Bio-Rad Universal Hood III, and analyzed by Image $\mathrm{Lab}^{\mathrm{TM}}$ software 2.0 (Bio-Rad).

Human Protein Atlas database. The expression of SGK3 in all major tissus and organs in the human body can be found in the Human Protein Atlas database. The proteins were detected by proteome methods (http://www.proteinatlas.org).

Statistical analysis. Data are presented as mean \pm standard deviation (SD) of one representative experiment. The differences in miR-144-3p expression between HCC tissues and non-cancerous tissues of human subjects were calculated by a two-tailed independent samples Student's t-test. Disease-free survival (DFS) was displayed by Kaplan-Meier survival curves, and DFS of different groups were compared by log-rank test. Unless otherwise noted, the differences between groups were analyzed by one-way analysis of variance (ANOVA) when there were more than two groups. In all cases, differences were considered statistically significant at $p<0.05$. All analyses were performed using SPSS16.0 software (Chicago, IL, USA).

\section{Results}

miR-144-3p expression in the HCC tissues and correlation with the clinicopathological features in HCC patients. In order to verify the expression of miR-144-3p in HCC, the levels of miR-144-3p in 51 paired HCC tissues were tested by qRT-PCR. As shown in Fig. 1A, miR-144-3p expression was significantly downregulated in $92 \%$ (47 of 51) of the HCC samples compared to their matched controls $(\mathrm{p}<0.001)$. We further found that low miR-144-3p expression was correlated with a shorter DFS $(\mathrm{p}<0.05)$ in the HCC patients as shown in Fig. 1B. These data suggested that miR-144-3p might be involved in tumor development and progression in HCC.
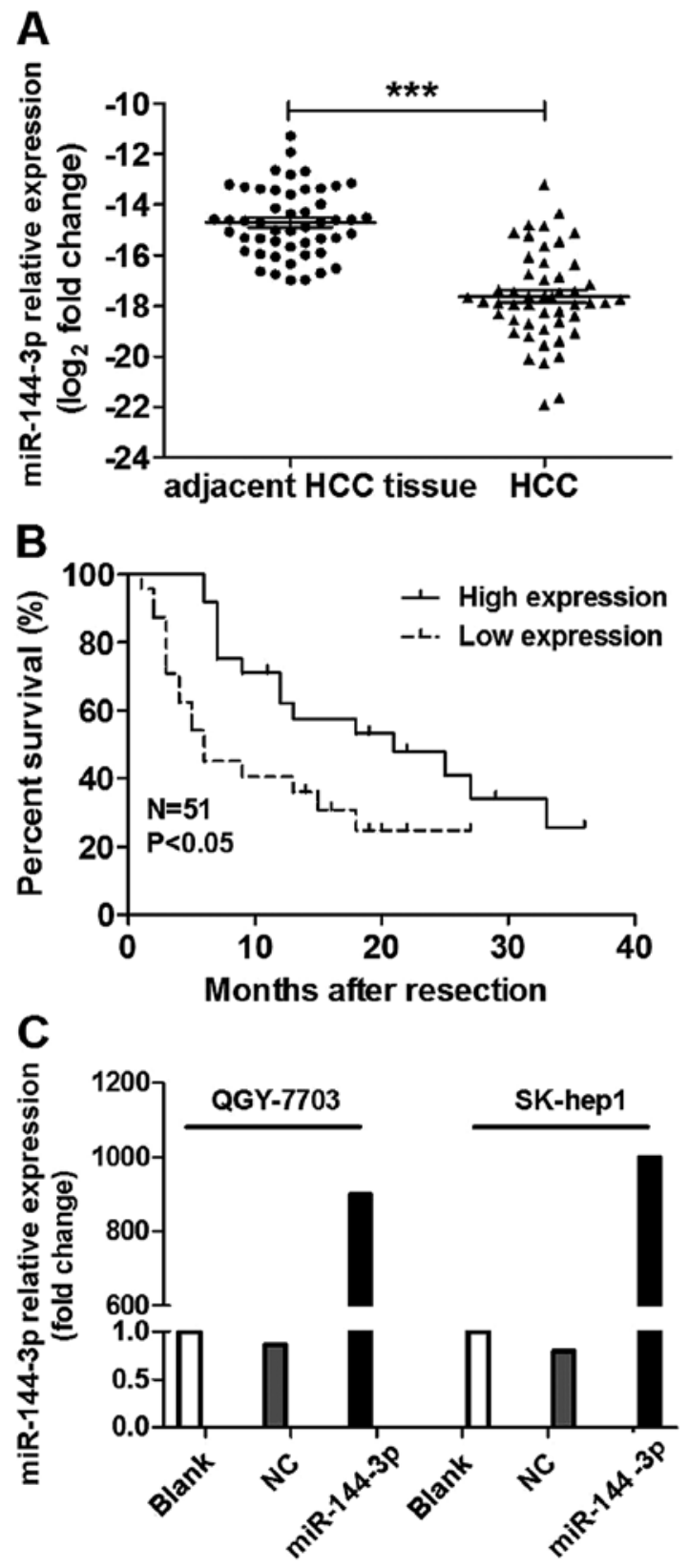

Figure 1. miR-144-3p expression was low in the HCC tissues and corresponded with survival time of HCC. (A) miR-144-3p expression levels in 51 paired HCC tissues and their matched controls were analyzed by qRT-PCR. (B) Patients with low miR-144-3p expression had poor DFS. Kaplan-Meier analyses of survival time in $51 \mathrm{HCC}$ patients according to the expression level of miR-144-3p. ${ }^{*} \mathrm{p}<0.05$. (C) miR-144-3p relative expression in the QGY-7703 and SK-hep1 after transfected with the miR-144-3p mimics or the NC duplex for 24 h. ${ }^{* * *} \mathrm{p}<0.001$.

Overexpression miR-144-3p inhibits proliferation and clonogenicity of HCC cells. To further reveal the effect of miR-144-3p expression in HCC cells. We used miRNA mimics to rebuilt the miR-144-3p expression, as shown in Fig. 1C. The expression of miR-144-3p in HCC cells was significantly improved after transfection with miR-144-3p mimics. The results of proliferation showed that improving miR-144-3p expression could significantly suppress the proliferation of QGY-7703 or SK-hep1 cells, respectively (Fig. 2A). The data on clonogenicity of HCC cells also found that QGY-7703 and SK-hep1 transfected with miR-144-3p mimic for 10 days 
A

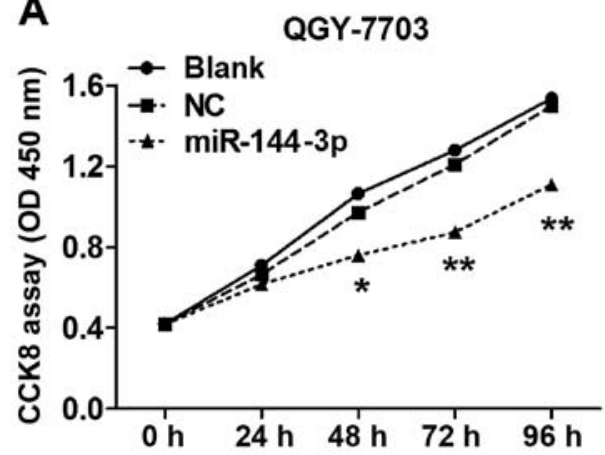

B
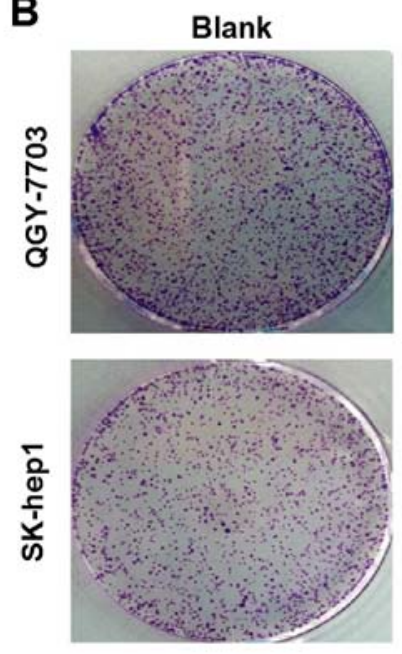

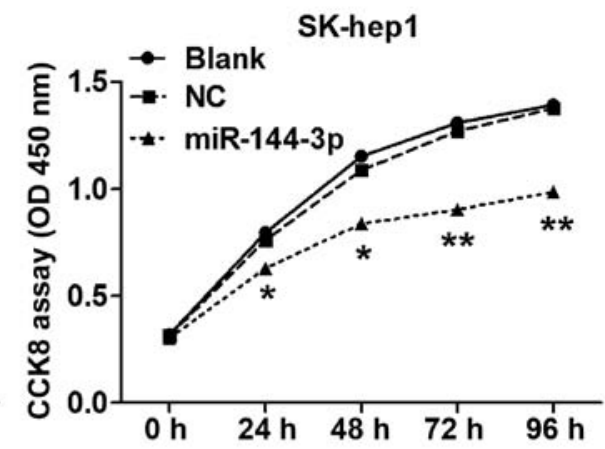

NC
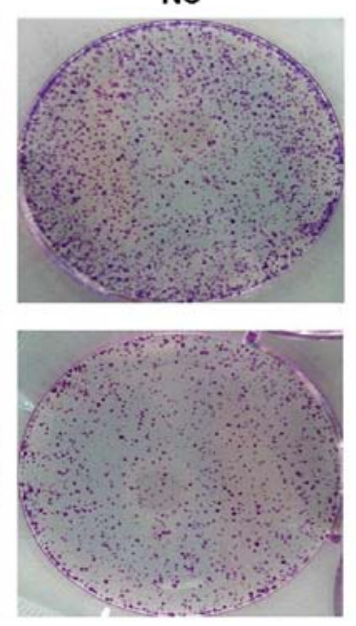

miR-144-3p
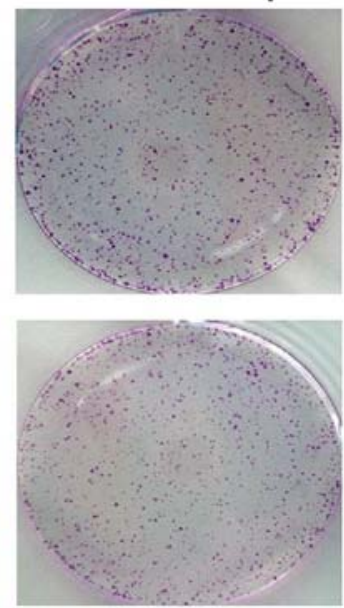

Figure 2. The effect of miR-144-3p overexpression on HCC cell proliferation and clonogenicity. (A) Cell proliferation of the HCC cell lines was evaluated using CCK-8 assay after transfection with the miR-144-3p mimics or NC at the indicated time. (B) Effect of miR-144-3p on the colony formation of the HCC cell lines. ${ }^{*} \mathrm{p}<0.05,{ }^{* *} \mathrm{p}<0.01$.

displayed notably fewer colonies compared with NC transfection (Fig. 2B) $(\mathrm{p}<0.05)$.

miR-144-3p suppresses HCC cell cycle progression to inhibit tumor growth. To determine whether the antitumor role of miR-144-3p correlates to HCC cell cycle progression, we analyzed cell cycle of QGY-7703 and SK-hep1 HCC cells treated with the miR-144-3p mimics for $48 \mathrm{~h}$ by flow cytometry. As observed in Fig. 3, miR-144-3p mimics significantly blocked G1 phase of QGY-7703 and SK-hep1 HCC cells compared to that of $\mathrm{NC}$. Therefore, the results indicated that miR-144-3p suppressed the cell cycle progression to inhibit the proliferation of HCC cells.

miR-144-3p overexpression inhibits HCC cell migration. The role of miR-144-3p on HCC cell migration was investigated. As revealed in Fig. 4, QGY-7703 and SK-hep1 cells with miR-144-3p restoration had significantly weaker migration than control cells $(\mathrm{P}<0.05)$. These observations implied that miR-144-3p could inhibit HCC metastasis.

miR-144-3p mimics reduce the ability of HCC cells to promote angiogenesis and tumor growth in vivo. In order to explore the function of miR-144-3p on the angiogenesis ability of HCC, HUVECs cultured on Matrigel in conditioned media from QGY-7703 cells and SK-hep1 cells transfected with miR-144-3p mimics formed fewer capillary-like rings than
HUVECs cultured in conditioned media from cells transfected with NC or siRNA as shown in the Fig. 5A. Moreover, the animal studies also showed that overexpression miR-144-3p suppressed the tumor growth in vivo compared to negative control (Fig. 5B). These results suggested that miR-144-3p could inhibit the ability of HCC cells to promote angiogenesis and tumor growth in vivo.

miR-144-3p inhibits HCC cell growth and angiogenesis PI3Kindependently by SKG3. To further explore the mechanism of miR-144-3p in tumor growth and angiogenesis of HCC, we found there were 1,043 putatively targeted genes of miR-144-3p in Target Scan. Then we used the DAVID Bioinformatics Resources 6.7 database to analyze the functional pathways (https://david-d.ncifcrf.gov), and found 8 types of rich pathways (Table I) after imputing these putative targets in the database, most pathways were shown involved in angiogenesis however, also relating to tumor growth process. Angiogenesis is a key process involved in tumor growth and metastasis. SGK3 has been shown to be highly expressed in HCC and is hardly expressed in normal liver tissue from the Human Protein Atlas database (Fig. 6A), so we aimed to identify the relationship between miR-144-3p and SGK3, and the effect of miR-144-3p targeting SKG3 in the inhibittion of PI3K/mTOR/ VEGF pathway activation. As shown in the Fig. 6B, we could find the sequence alignment of miR-144-3p and its conserved target position in SKG3 3'UTR where miR-144-3p might 


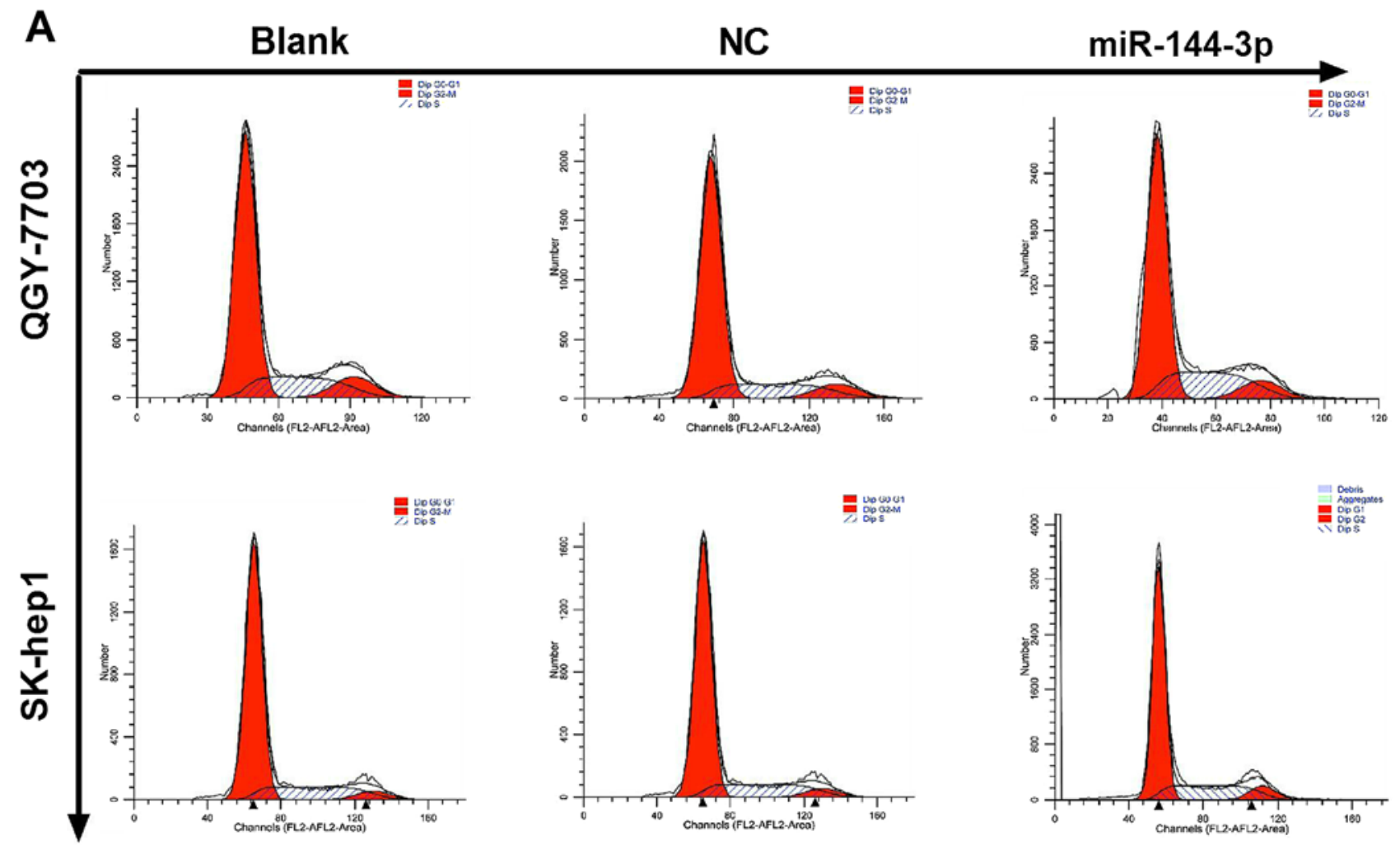

B

QGY-7703
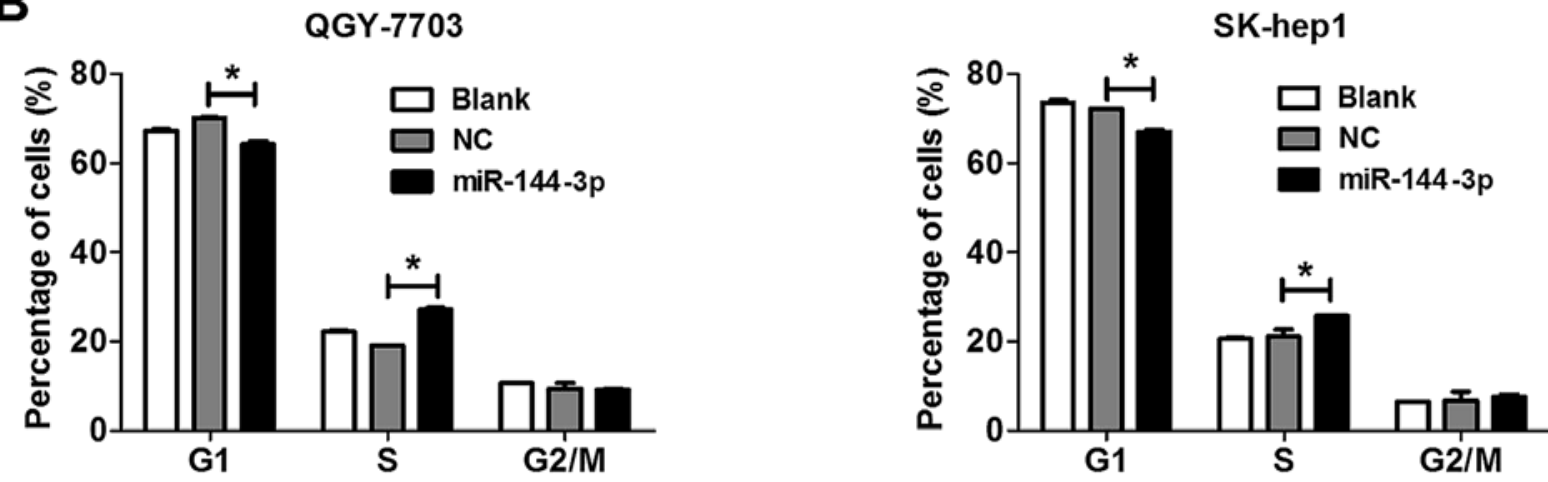

Figure 3. miR-144-3p suppresses HCC cell cycle progression to inhibit tumor growth. (A) QGY-7703 and SK-hep1 cells, which were transfected with miR-144-3p mimics or NC for $48 \mathrm{~h}$, were harvested and analyzed by FACS and (B) the cell cycle distribution was determined. Data are shown as the mean $\pm \mathrm{SD}(\mathrm{n}=4)$ of one representative experiment. Similar results were obtained in three independent experiments. ${ }^{*} \mathrm{p}<0.05$.

Table I. Eight types of rich pathways has been found by the DAVID Bioinformatics Resources 6.7 database (https://david-d. ncifcrf.gov).

\begin{tabular}{lllrrr}
\hline Category & \multicolumn{1}{c}{ Term } & RT & Count & $\%$ & P-value \\
\hline PANTHER_PATHWAY & P00021:FGF signaling pathway & RT & 19 & 0.2 & $7.6 \mathrm{E}-3$ \\
PANTHER_PATHWAY & P00047:PDGF signaling pathway & RT & 22 & 0.2 & $2.1 \mathrm{E}-2$ \\
PANTHER_PATHWAY & P00057:Wnt signaling pathway & RT & 35 & 0.3 & $2.3 \mathrm{E}-2$ \\
PANTHER_PATHWAY & P00018:EGF receptor signaling pathway & RT & 18 & 0.2 & $2.6 \mathrm{E}-2$ \\
PANTHER_PATHWAY & P00005:Angiogenesis & RT & 25 & 0.2 & $3.0 \mathrm{E}-2$ \\
PANTHER_PATHWAY & P00034:Integrin signaling pathway & RT & 24 & 0.2 & $4.5 \mathrm{E}-2$ \\
PANTHER_PATHWAY & P00033:Insulin/IGF pathway-protein kinase B signaling cascade & RT & 12 & 0.1 & $5.3 \mathrm{E}-2$ \\
PANTHER_PATHWAY & P00035:Interferon- $\gamma$ signaling pathway & RT & 6 & 0.1 & $8.9 \mathrm{E}-2$ \\
\hline
\end{tabular}

Term, Enriched Functional Annotation; RT, related terms; P-value, enrichment P-values; FGF, fibroblast growth factor; PDGF, platelet-derived growth factor; EGF, epidermal growth factor; \%, percentage. 
A
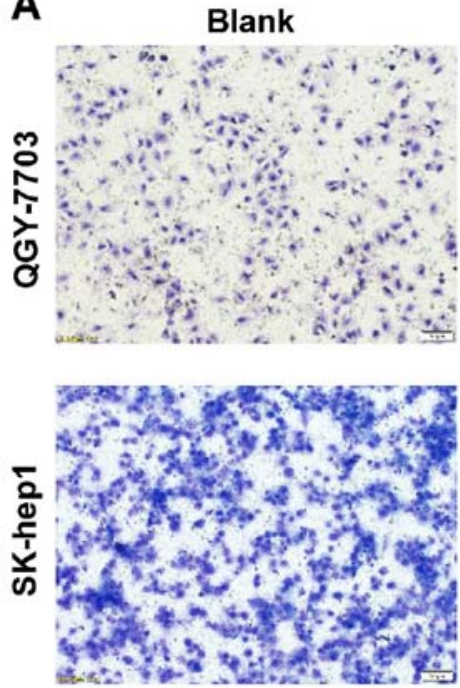

B

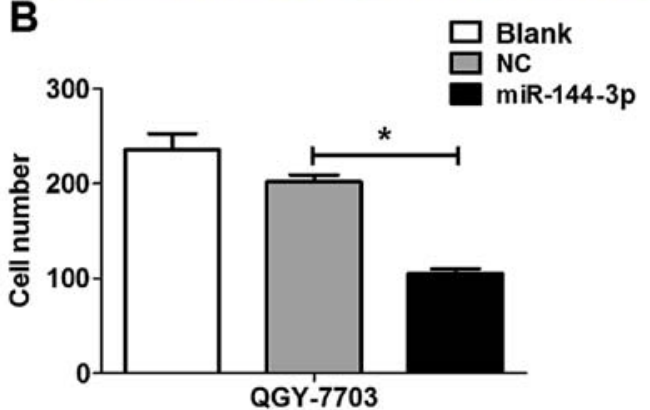

NC
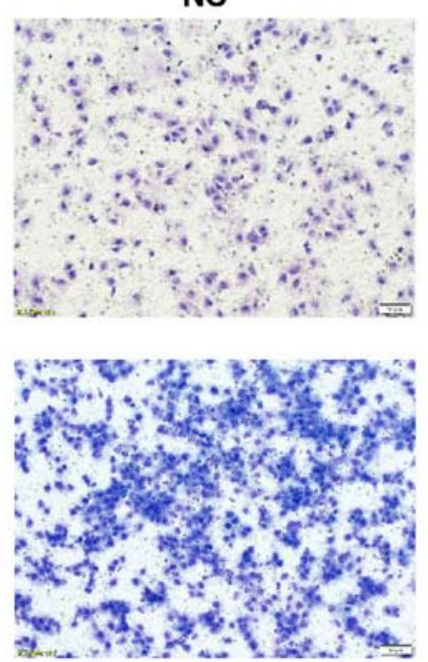
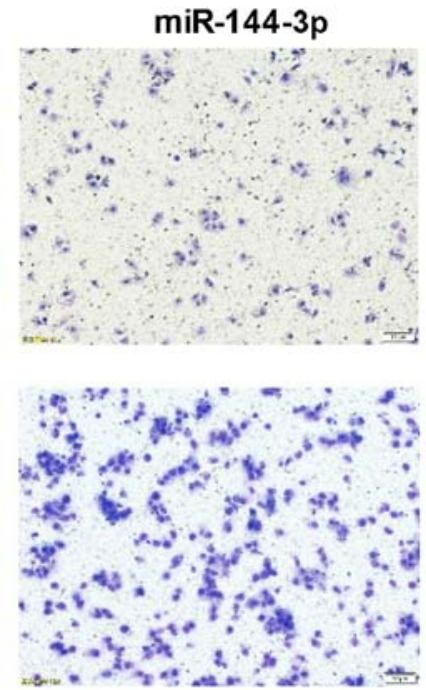

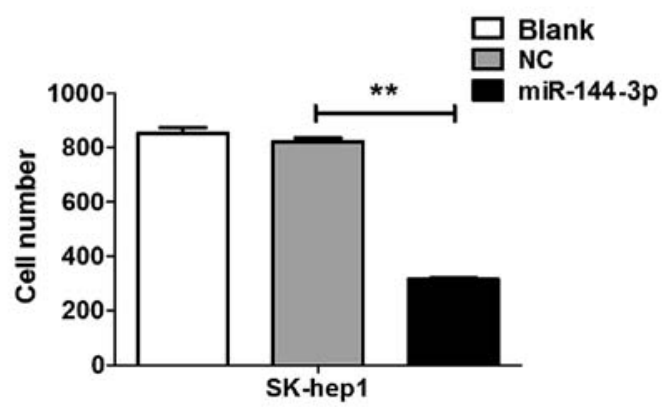

Figure 4. miR-144-3p inhibits cell migration in HCC cells. (A) Transwell migration assays in the QGY-7703 and SK-hep1 cells transfected with miR-144-3p mimics or NC. (B) The number of cells was calculated with crystal violet staining. Data are shown as mean \pm SD ( $\mathrm{n}=4$ ) of one representative experiment. Similar results were obtained in three independent experiments. ${ }^{*} \mathrm{p}<0.05,{ }^{* *} \mathrm{p}<0.01$. NC, negative control.

B

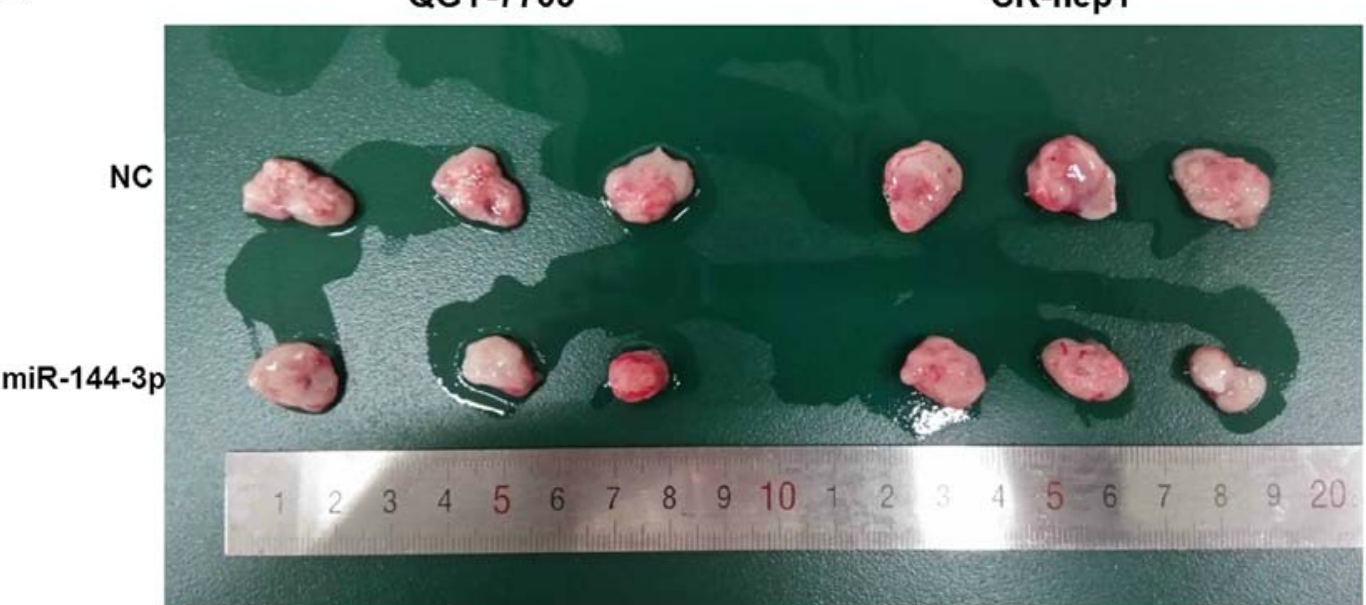

A

QGY-7703-miR-144

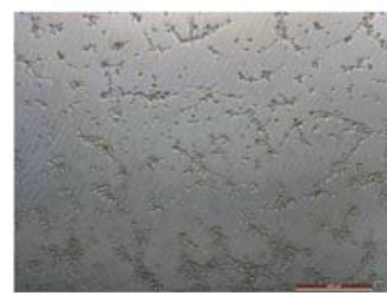

$24 \mathrm{~h}$

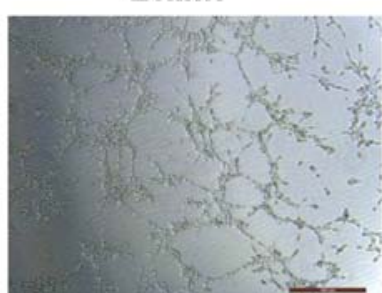

QGY-7703

\section{SK-hep1-miR-144-3p}

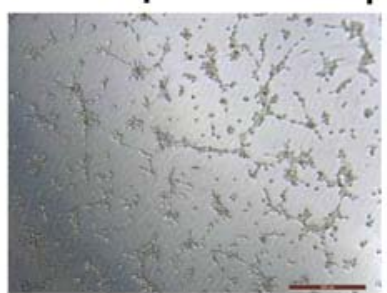

SK-hep1 


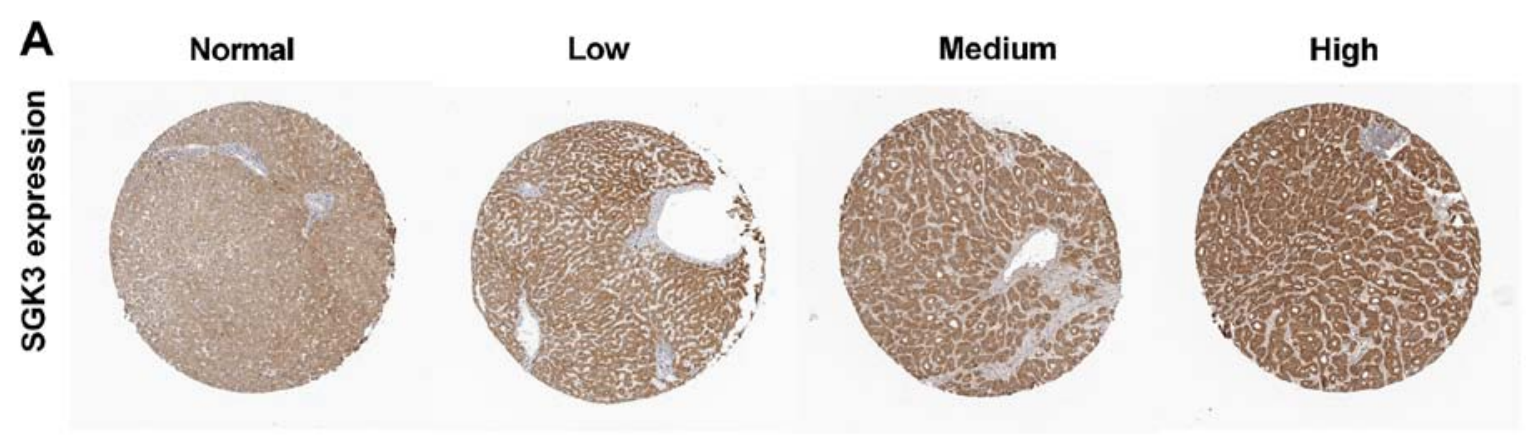

\section{B Position 2221-2227 of SGK3 3' UTR: 5'...UGGUUUAUUUGGCACUACUGUAA...3' IIIIIII hsa-miR-144-3p: UCAUGUAGUAGAUAUGACAU}

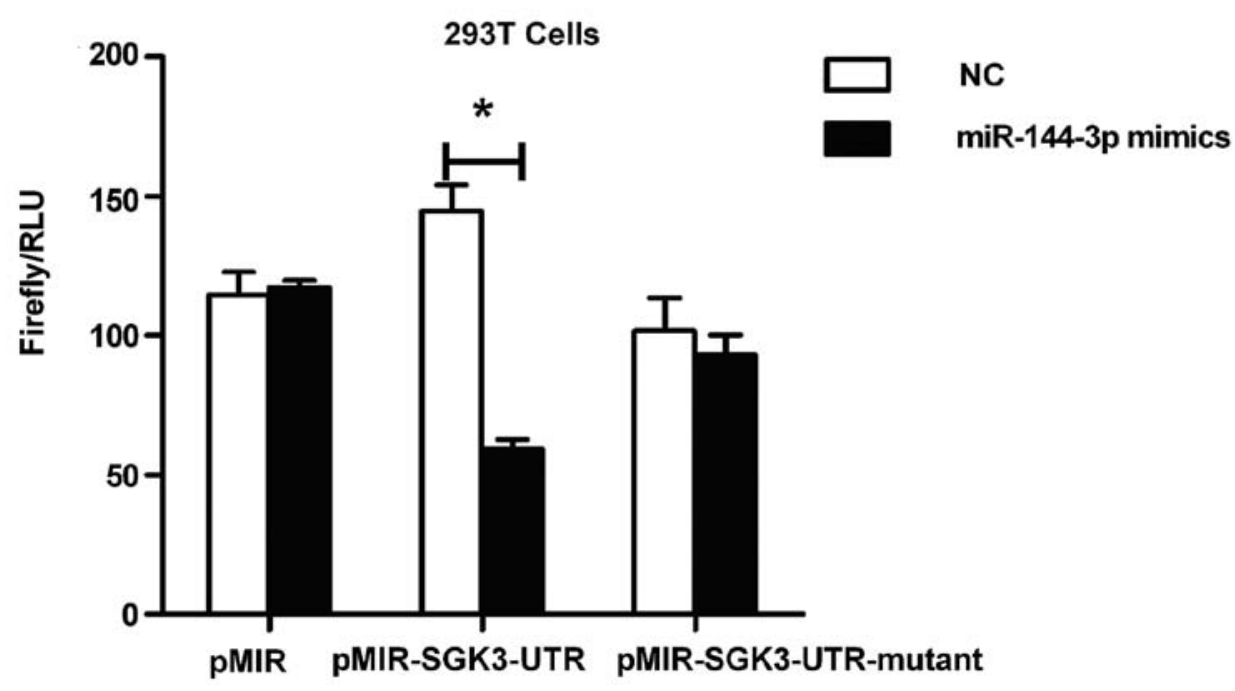

Figure 6. miR-144-3p directly targets SGK3. (A) SGK3 has high expression in HCC while it is hardly expressed in normal liver tissue based on Human Protein Atlas database. (B) Sequence alignment of miR-144-3p and its conserved target position in SKG3-3'UTR. miR-144-3p significantly inhibited the activity of the firefly luciferase that carried wild-type, but not the mutant 3'UTR of SGK3.

suppress gene expression through its binding sequence. As observed in Fig. 7A, the mRNA of mTOR and VEGF2 were significantly downregulated by miR-144-3p mimics after targeting SGK3, but the mRNA of PI3K showed no obvious change in HCC cells after transfected with miR-144-3p mimics. Similar to the function of SGK3-siRNA, the results of WB found that overexpression of miR-144-3p not only suppressed the phosphorylation of PI3K, but also repressed the protein expression of mTOR and VEGFR2, which are in PI3K downstream signal pathway in HCC cells (Fig. 7B). These results demonstrated that miR-144-3p could suppress tumor growth and angiogenesis by targeting SGK3 with $\mathrm{PI} 3 \mathrm{~K} / \mathrm{SGK} / \mathrm{mTOR}$ pathway.

\section{Discussion}

miRNAs have been indicated to play an important role in diverse biological processes, including cancer. Emerging evidence has demonstrated aberrant expression of miRNAs involving in the tumor development and metastasis process. Thus, revealing the potential mechanism of miRNAs in cancer can promote our understanding in cancer biology and offer new knowledge to invent new treatments and diagnosis methods (14).
miR-144-3p, as a tumor suppressor, has been demonstrated in several cancer types. For instance, Navon et al (15) using microarrays profiled the expression of $>700$ miRNAs in 28 matched tumor and normal samples representing 32 microarray measurements from 8 different tumor types (breast, colon, liver, lung, lymphoma, ovary, prostate and testis). In addition, there are some findings that miR-144-3p inhibits proliferation and induces apoptosis and autophagy in lung cancer cells by targeting TIGAR (16); and miR-144-3p downregulation increases bladder cancer cell proliferation by targeting EZH2 and regulating Wnt signaling (17). On the contrary, Zhang et al reported that miR-144-3p could promote tumor cell proliferation, migration and invasion in nasopharyngeal carcinoma through repression of PTEN (18), and Liu et al found that Myc induced miR-144-3p/451 contributing to the acquired imatinib resistance in chronic myelogenous leukemia K562 cells (19). In this study, we found that miR-144-3p acted as a tumor suppressor, which is downregulated in HCC, recovery of the expression of miR-144-3p was able to significantly inhibit HCC growth, migrations and angiogenesis. Also, the clinical data showed that high expression of miR-144-3p was correlated with long disease-free survival time. This result showed that miR-144-3p could be a prognostic marker for HCC. 
A

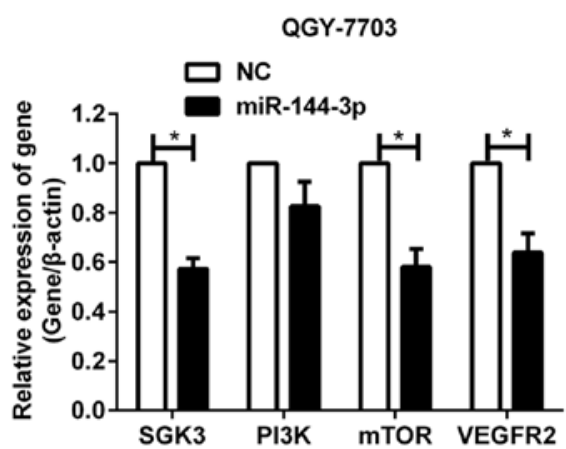

B

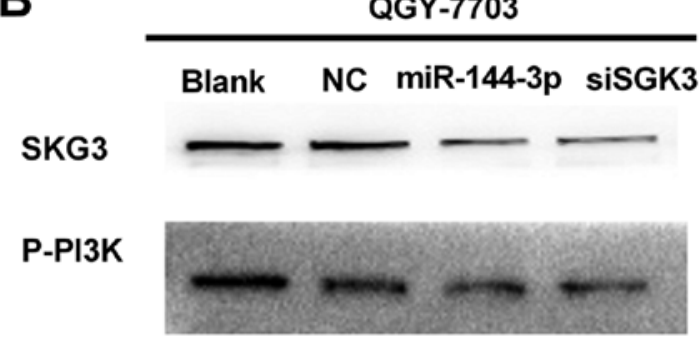

mTOR

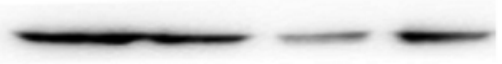

VEGFR2

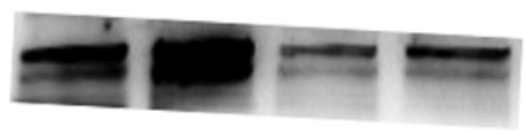

$\beta$-actin
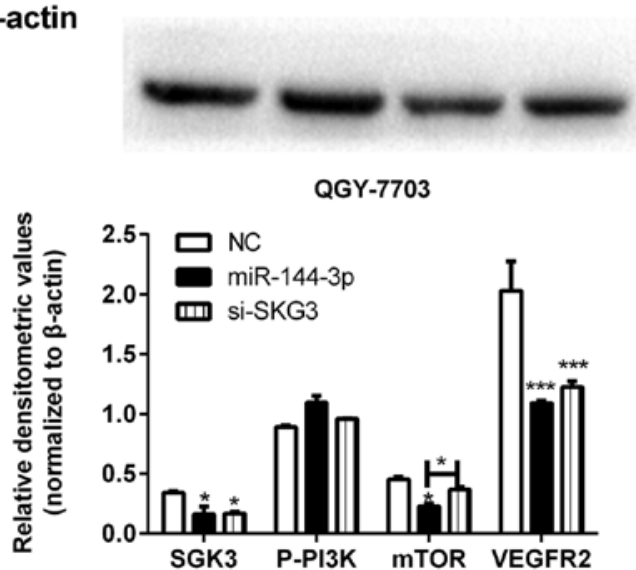

QGY-7703

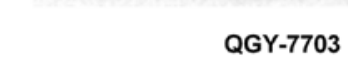

言

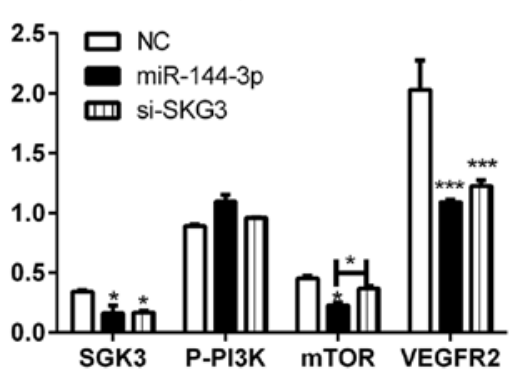

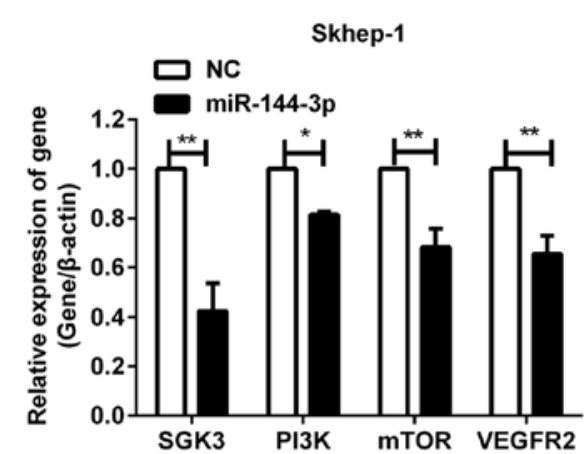

SKhep-1
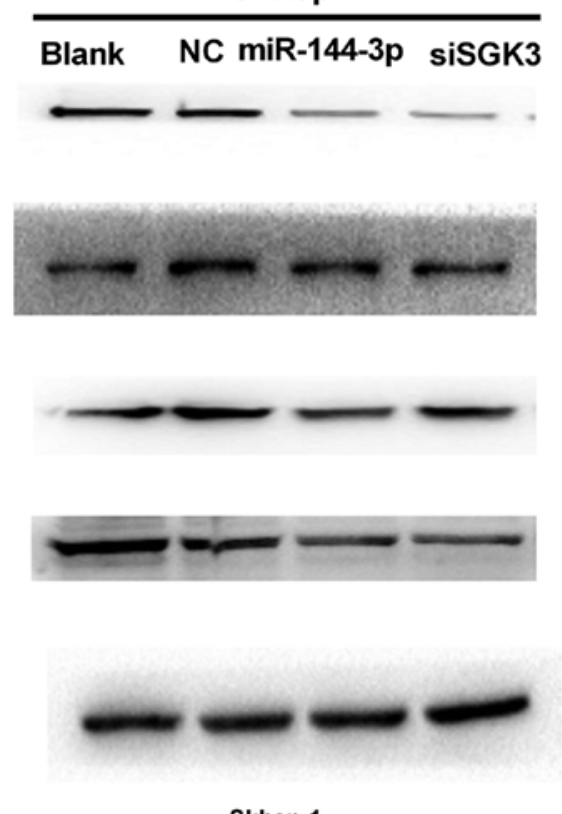

Skhep-1

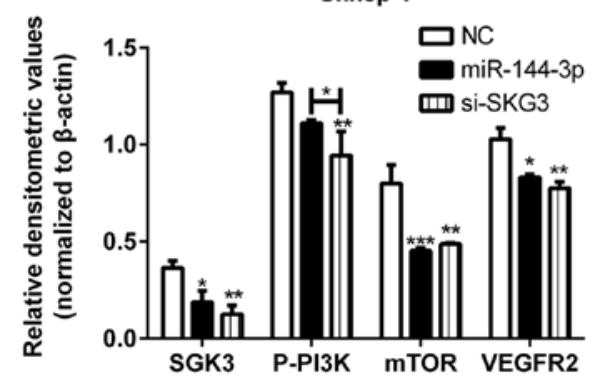

Figure 7. miR-144-3p inhibits PI3K-AKT pathway activation by targeting SGK3. (A) The mRNA of SKG3, PI3K, mTOR and VEGFR2 in the QGY-7703 and SK-hep1 cells were analyzed by qRT-PCR. (B) Protein expression of the PI3K/SKG3/mTOR pathway members were detected in the QGY-7703 and SK-hep1 cells after treatment with miR-144-3p or NC for $24 \mathrm{~h}$. Data are shown as mean \pm SD $(n=4)$ of one representative experiment.

To better understand the mechanisms of miR-144-3p on HCC, we used Target-Scan database and DAVID Bioinformatics Resources 6.7 database to analyze the potential targets and relative function pathways. The results (Table I), showed most targets were correlated with angiogenesis and tumor growth. As angiogenesis has been verified to promote tumor growth, metastasis and even cause resistance (20-26), we chose SGK3 as potential target of miR-144-3p to investigate whether SGK3 can influence tumor angiogenesis and growth in HCC. Encoded by chromosome $8 \mathrm{q} 12.2$, SGK is known as a downstream mediator of phosphatidylinositol 3-kinase (PI3K) oncogenic signaling. It has also been proved that SGK3 plays a pivotal role in oncogenic progress in various cancers, including breast cancer, ovarian cancer and hepatocellular carcinoma (27). In physiological research aspect, some studies reported that SGK1 is necessary for vascular remodeling during angiogenesis (28), and ablation of SGK1 impairs endothelial cell migration and tube formation leading to decreased neo-angiogenesis following myocardial infarction (29). Thus, we hypothesized that miR-144-3p might target SGK3 to inhibit tumor growth and angiogenesis. Consistent with similar studies, we found that miR-144-3p decreased the activation of mTOR and VEGF with no obvious change of PI3K, and displayed more inhibitor effect on mTOR and VEGFR2 expression in HCC cells, compared with the SGK3-siRNA groups. These results indicated that miR-144-3p has powerful antitumor function. 
In conclusion, we found miR-144-3p, similarly to a tumor suppressor, could be a prognostic factor and inhibit tumor growth and angiogenesis by targeting SGK3 suppressing activation of mTOR/VEGFR2 of PI3K downstream signals. However, regardless of the role of miR-144-3p in drugresistance or metastasis, more in vitro and in vivo studies are needed to fully reveal the downregulation of miR-144-3p and its underlying mechanisms.

\section{Acknowledgements}

This study was supported by grants from National Natural Science Foundation of China (no. 81560493), Key Project of Natural Science Foundation of Guangxi (no. 2015GXNSFDA139024) and the Research Fund for the College Scientific Program of Education Department of Guangxi Province (no. ZD2014027).

\section{References}

1. Siegel RL, Miller KD and Jemal A: Cancer statistics, 2016. CA Cancer J Clin 66: 7-30, 2016.

2. Aravalli RN, Steer CJ and Cressman EN: Molecular mechanisms of hepatocellular carcinoma. Hepatology 48: 2047-2063, 2008.

3. Aravalli RN, Cressman EN and Steer CJ: Cellular and molecular mechanisms of hepatocellular carcinoma: An update. Arch Toxicol 87: 227-247, 2013.

4. Kong YW, Ferland-McCollough D, Jackson TJ and Bushell M: microRNAs in cancer management. Lancet Oncol 13: e249-e258, 2012.

5. Zhou X, Zhang CZ, Lu SX, Chen GG, Li LZ, Liu LL, Yi C, Fu J, $\mathrm{Hu} \mathrm{W}$, Wen JM, et al: miR-625 suppresses tumour migration and invasion by targeting IGF2BP1 in hepatocellular carcinoma. Oncogene 34: 965-977, 2015

6. Hou J, Lin L, Zhou W, Wang Z, Ding G, Dong Q, Qin L, Wu X, Zheng Y, Yang Y, et al: Identification of miRNomes in human liver and hepatocellular carcinoma reveals miR-199a/b-3p as therapeutic target for hepatocellular carcinoma. Cancer Cell 19: 232-243, 2011.

7. Guan H, Liang W, Xie Z, Li H, Liu J, Liu L, Xiu L and Li Y: Down-regulation of miR-144 promotes thyroid cancer cell invasion by targeting ZEB1 and ZEB2. Endocrine 48: 566-574, 2015.

8. Zha W, Cao L, Shen Y and Huang M: Roles of MiR-144-ZFX pathway in growth regulation of non-small-cell lung cancer. PLoS One 8: e74175, 2013.

9. Huang XP, Hou J, Shen XY, Huang CY, Zhang XH, Xie YA and Luo XL: MicroRNA-486-5p, which is downregulated in hepatocellular carcinoma, suppresses tumor growth by targeting PIK3R1. FEBS J 282: 579-594, 2015.

10. Bruhn MA, Pearson RB, Hannan RD and Sheppard KE: AKT-independent PI3-K signaling in cancer - emerging role for SGK3. Cancer Manag Res 5: 281-292, 2013.

11. Cao T, Li H, Hu Y, Ma D and Cai X: miR-144 suppresses the proliferation and metastasis of hepatocellular carcinoma by targeting E2F3. Tumour Biol 35: 10759-10764, 2014.

12. Wang R, Zhao N, Li S, Fang JH, Chen MX, Yang J, Jia WH, Yuan $Y$ and Zhuang SM: MicroRNA-195 suppresses angiogenesis and metastasis of hepatocellular carcinoma by inhibiting the expression of VEGF, VAV2, and CDC42. Hepatology 58: 642-653, 2013
13. Yang X, Zhang XF, Lu X, Jia HL, Liang L, Dong QZ, Ye QH and Qin LX: MicroRNA-26a suppresses angiogenesis in human hepatocellular carcinoma by targeting hepatocyte growth factor-cMet pathway. Hepatology 59: 1874-1885, 2014.

14. Zhu Z, Zhang X, Wang $G$ and Zheng $H$ : Role of microRNAs in hepatocellular carcinoma. Hepat Mon 14: e18672, 2014.

15. Navon R, Wang H, Steinfeld I, Tsalenko A, Ben-Dor A and Yakhini Z: Novel rank-based statistical methods reveal microRNAs with differential expression in multiple cancer types. PLoS One 4: e8003, 2009.

16. Chen S, Li P, Li J, Wang Y, Du Y, Chen X, Zang W, Wang H, Chu H, Zhao G, et al: MiR-144 inhibits proliferation and induces apoptosis and autophagy in lung cancer cells by targeting TIGAR. Cell Physiol Biochem 35: 997-1007, 2015.

17. Guo Y, Ying L, Tian Y, Yang P, Zhu Y, Wang Z, Qiu F and Lin J: miR-144 downregulation increases bladder cancer cell proliferation by targeting EZH2 and regulating Wnt signaling. FEBS J 280: 4531-4538, 2013.

18. Zhang LY, Ho-Fun Lee V, Wong AM, Kwong DL, Zhu YH, Dong SS, Kong KL, Chen J, Tsao SW, Guan XY, et al: MicroRNA-144 promotes cell proliferation, migration and invasion in nasopharyngeal carcinoma through repression of PTEN. Carcinogenesis 34: 454-463, 2013.

19. Liu L, Wang S, Chen R, Wu Y, Zhang B, Huang S, Zhang J, Xiao F, Wang M and Liang Y: Myc induced miR-144/451 contributes to the acquired imatinib resistance in chronic myelogenous leukemia cell K562. Biochem Biophys Res Commun 425: 368-373, 2012

20. Yuan SX, Yang F, Yang Y, Tao QF, Zhang J, Huang G, Yang Y, Wang RY, Yang S, Huo XS, et al: Long noncoding RNA associated with microvascular invasion in hepatocellular carcinoma promotes angiogenesis and serves as a predictor for hepatocellular carcinoma patients' poor recurrence-free survival after hepatectomy. Hepatology 56: 2231-2241, 2012.

21. Fang JH, Zhou HC, Zeng C, Yang J, Liu Y, Huang X, Zhang JP Guan XY and Zhuang SM: MicroRNA-29b suppresses tumor angiogenesis, invasion, and metastasis by regulating matrix metalloproteinase 2 expression. Hepatology 54: 1729-1740, 2011.

22. Fernández M, Semela D, Bruix J, Colle I, Pinzani M and Bosch J: Angiogenesis in liver disease. J Hepatol 50: 604-620, 2009.

23. Thomas MB, Jaffe D, Choti MM, Belghiti J, Curley S, Fong Y, Gores G, Kerlan R, Merle P, O'Neil B, et al: Hepatocellular carcinoma: Consensus recommendations of the National Cancer Institute Clinical Trials Planning Meeting. J Clin Oncol 28: 3994-4005, 2010.

24. Hao CY: Angiogenesis blockade as therapy for hepatocellular carcinoma: Progress and challenges. J Gastroenterol Hepatol 26: 4-6, 2011.

25. Zhang L, Wang JN, Tang JM, Kong X, Yang JY, Zheng F, Guo LY, Huang YZ, Zhang L, Tian L, et al: VEGF is essential for the growth and migration of human hepatocellular carcinoma cells. Mol Biol Rep 39: 5085-5093, 2012.

26. Kerbel RS: Tumor angiogenesis. N Engl J Med 358: 2039-2049, 2008.

27. Hou M, Lai Y, He S, He W, Shen H and Ke Z: SGK3 (CISK) may induce tumor angiogenesis (Hypothesis). Oncol Lett 10: 23-26, 2015.

28. Catela C, Kratsios P, Hede M, Lang F and Rosenthal N: Serum and glucocorticoid-inducible kinase 1 (SGK1) is necessary for vascular remodeling during angiogenesis. Dev Dyn 239: 2149-2160, 2010.

29. Zarrinpashneh E, Poggioli T, Sarathchandra P, Lexow J, Monassier L, Terracciano C, Lang F, Damilano F, Zhou JQ, Rosenzweig A, et al: Ablation of SGK1 impairs endothelial cell migration and tube formation leading to decreased neo-angiogenesis following myocardial infarction. PLoS One 8: e80268, 2013. 